\title{
Renal Denervation Attenuates Multi-Organ Fibrosis and Improves Vascular Remodeling in Rats with Transverse Aortic Constriction Induced Cardiomyopathy
}

\author{
Kai Wang Dasheng Lu Bin Zhang Shengchan Wang Qian Liu Qi Zhang \\ Jie Geng Qijun Shan
}

Department of Cardiology, The First Affiliated Hospital of Nanjing Medical University, Nanjing, P.R. China

\section{Key Words}

Renal Denervation • Carvedilol • Heart Failure • Fibrosis $•$ Vascular Remodeling

\begin{abstract}
Background/Aims: To investigate the effects of renal denervation (RDN) on multi-organ fibrosis and vascular remodeling in cardiomyopathy. Methods: Thirty-six male Sprague-Dawley rats underwent transverse aortic constriction (TAC). Five weeks later, 28 surviving TAC rats were randomly assigned to three groups: (1) RDN, (2) Sham, (3) Carvedilol. Six male Sham TAC rats served as the Control. Ten weeks after TAC, samples were collected. Results: TAC rats showed an increased diastolic interventricular septal thickness at week 5. At 10 weeks, Masson staining showed that left ventricular and renal glomerular fibrosis were significantly reduced in RDN compared with Sham group. In comparison to Sham group, hepatic perivascular fibrosis was attenuated in both RDN and Carvedilol group, so were the media thickness and the media/ lumen of aorta. The plasma levels of B-type natriuretic peptide (BNP), Cystatin C (Cys-C), Alanine Transaminase, angiotensin II (Ang II), transforming growth factor beta 1 (TGF- $\beta 1$ ), and malondialdehyde increased, and total superoxide dismutase (T-SOD) decreased in Sham but not in RDN group, compared with Control group. Both RDN and Carvedilol reduced the Cys-C and TGF- $\beta 1$ levels, and restored T-SOD concentration, compared with Sham group. While only RDN lowered the plasma levels of BNP and Ang II. No significant effects of RDN on blood pressure (BP) and heart rate (HR) were oberved. Conclusions: RDN can attenuate multi-organ fibrosis and improve vascular remodeling independent of BP and HR change in TAC-induced cardiomyopathy. These effects of RDN may be associated with the direct inhibition of reninangiotensin-aldosterone system and oxidative stress.
\end{abstract}

K. Wang and D. Lu contributed equally to the work. 


\begin{tabular}{|c|c|}
\hline Cellular Physiology & Cell Physiol Biochem 2016;40:465-476 \\
\hline and Binch & 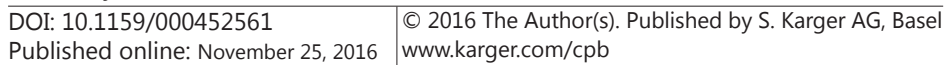 \\
\hline
\end{tabular}

\section{Introduction}

Cardiac pressure overload leads to maladaptive myocardial remodeling, including cardiac hypertrophy, systolic and diastolic dysfunction as well as fibrosis [1, 2]. Cardiac dysfunction can cause kidney and liver damage $[3,4]$. Also, there is a crosstalk between heart and kidney [3]. Cardiac and renal dysfunction and fibrosis amplify the risks of failure and fibrosis of other organs, including the liver, due to inflammatory factors, reactive oxygen species and the over activity of sympathetic nervous system and the renin-angiotensinaldosterone system (RAAS) [3,5]. Fibrosis, which occurs as a result of the proliferation of interstitial fibroblasts and deposition of extracellular matrix [6], may be the common pathway for multi-organ damage. Fibrosis in the heart leads to various cardiac disorders, such as heart failure (HF) and cardiac arrhythmias [7]. Fibrosis in kidney results in chronic kidney disease [8]. Fibrosis in liver accounts for cirrhosis and hepatocellular carcinoma [9]. Simultaneously, vascular remodeling may lead to arteriosclerosis [10]. It is an urgent problem to prevent multi-organ fibrosis and vascular remodeling in HF.

Renal denervation (RDN) represents a novel technique, which can reduce blood pressure (BP) and heart rate (HR) in patients with resistant hypertension [11, 12]. Apart from the beneficial effects on BP, RDN can also improve left ventricular hypertrophy and diastolic function [13]. The mechanism may be associated with the inhibition of fibrosis [14]. However, effects of RDN on multi-organ fibrosis and vascular remodeling in HF are little known. The aim of this study was to investigate the effects of RDN on multi-organ fibrosis and vascular remodeling in cardiomyopathy and compare its effects with carvedilol.

\section{Materials and Methods}

Animals and experimental design

All procedures in this study were approved by the Ethics Committee of Nanjing Medical University. The animal experiments were performed conform the NIH guidelines (Guide for the care and use of laboratory animals) and the guidelines from Directive 2010/63/EU of the European Parliament on the protection of animals used for scientific purposes. Forty-two six-week old Sprague-Dawley rats (200 - $220 \mathrm{~g}$ ) were provided by Nanjing Medical University Laboratory Animal Center. After being housed in cages for one week for adaption period, 36 rats underwent transverse aortic constriction (TAC) procedure, while 6 rats underwent sham TAC served as Control. Echocardiography was performed at week $0,5,10$. TAC and RDN were performed after echocardiography at week 0 and 5, respectively. At week 5, 28 surviving TAC rats were randomly assigned to 3 groups: TAC with sham RDN operation (Sham, $\mathrm{n}=10$ ), TAC with carvedilol (Carvedilol, $\mathrm{n}=9$ ) and TAC with RDN operation (RDN, $\mathrm{n}=9$ ). Carvedilol was given orally at a dose of $10 \mathrm{mg}$ / $\mathrm{kg}$ for 5 weeks. The dosing regimen of carvedilol was based on previous study [15].

\section{Transverse aortic constriction}

TAC was performed in rats as described previously [16]. In brief, rats were anesthetized with a single intraperitoneal injection of pentobarbital sodium $(60 \mathrm{mg} / \mathrm{kg})$ until they lost the pedal withdrawal reflex. Then, during ventilation with $100 \%$ oxygen, thoracotomy was performed at the second intercostal space, and the ascending aorta was partially occluded using a bent 20-Gauge needle. Then, the needle was quickly removed after ligation. Rats in the Control group experienced the same procedures, but aortic arch was not ligated.

\section{Renal denervation}

Five weeks after TAC, bilateral RDN or sham operation was performed in rats. The surgical procedures were performed as described previously [17]. In brief, rats were anesthetized with intraperitoneal injection of pentobarbital sodium $(60 \mathrm{mg} / \mathrm{kg}$ ). The loss of the pedal withdrawal reflex was regarded as the adequacy of anesthesia. Then, all visible nerves, connective tissue and fat around the renal arteries were stripped or severed. Next, renal vessels were painted with $20 \%$ phenol in an alcohol solution to destroy the remaining nerves. Finally, renal arteries were washed with isotonic saline. The Control, Sham and Carvedilol groups underwent the same procedures, but the renal vessels and nerves were not handled. 


\section{Cellular Physiology Cell Physiol Biochem 2016;40:465-476 \begin{tabular}{l|l|l} 
and BiOChemistry $\begin{array}{l}\text { DOI: 10.1159/000452561 } \\
\text { Published online: November 25, } 2016\end{array}$ & $\begin{array}{l}\text { (c) 2016 The Author(s). Published by S. Karger AG, Basel } \\
\text { www.karger.com/cpb }\end{array}$
\end{tabular} \\ Wang et al.: Renal Denervation and Multi-Organ Fibrosis}

\section{Echocardiography and hemodynamics}

Echocardiography was performed under anesthesia with isoflurane with Vevo2100 (VisualSonics, Canada) system equipped with a MS-250, 16.0-21.0 MHZ imaging transducer. HR was recorded at the same time. Tail-cuff pressure was measured 5 weeks after RDN in conscious state by non-invasive method using the Coda system (Coda, Kent Scientific, USA).

\section{Histological analysis}

All rats were sacrificed after being injected with an overdose of pentobarbital sodium (200 mg/kg). Then, the heart, kidneys, liver, and aorta were taken out from the body respectively after perfusion with icecold PBS. The excised hearts were separated into left atrium (LA) and left ventricular (LV) and fixed with $4 \%$ paraformaldehyde and then embedded in paraffin. For other organs, the procedures were the same. Masson's trichrome staining was performed to detect LA, LV, kidney and liver fibrosis as well as vascular remodeling. We randomly selected 6 microscopic fields (X 400) for analysis. Collagen volume fraction (CVF), expressed as the percentage of pixels of positive collagen staining divided by total pixels of per field was used to evaluate the extents of fibrosis by the Image-Pro Plus 6.0. The media thickness, the lumen diameter and the media/lumen of the aorta, indexes of vascular remodeling [18] were also analyzed by this software.

\section{Immunohistochemistry}

Immunohistochemical staining of kidney for tyrosine hydroxylase (TH) was performed using antityrosine hydroxylase antibody. Six representative fields were randomly selected for analysis and the integrated optical density per field (IOD) was measured by Image-Pro Plus 6.0.

\section{Enzyme linked immunosorbent assay}

Blood samples were collected into tubes containing EDTA, and then centrifuged at $3000 \mathrm{rpm}$ at $4^{\circ} \mathrm{C}$ for 10 min to separate the plasma, which was stored at $-80^{\circ} \mathrm{C}$ until enzyme linked immunosorbent assay (ELISA) testing. Corresponding ELISA kits (Nanjing Jiancheng Biological Product, Nanjing, China) were prepared to analyze the activity of total superoxide dismutase (T-SOD). Kidneys specimen were homogenized and centrifuged at $3000 \mathrm{rpm}$ for $10 \mathrm{~min}$ and then the supernatant was collected. Renal norepinephrine (NE) content was measured to verify the efficacy of RDN by ELISA Kit (E-EL-0047c, Elabscience Biotechnology, Wuhan, China). Cystatin C (Cys-C) and Alanine Transaminase (ALT) in plasma were measured using Cys-C ELISA kit(E-EL-R0304c, Elabscience Biotechnology, Wuhan, China) and ALT ELISA kit(E-EL-R1232c, Elabscience Biotechnology, Wuhan, China), respectively. Plasma B-type natriuretic peptide (BNP), angiotensin II (Ang II), amino-terminal pro-peptides of types I and III collagen (PINP and PIIINP), transforming growth factor beta 1 (TGF- $\beta 1$ ), norepinephrine (NE) and malondialdehyde (MDA) levels were tested using ELISA kits according to the instruction books (Uscn Life Science Inc, Wuhan, China) at week 10 .

\section{Statistical analysis}

All data are shown as mean \pm SEM. Statistical analysis was performed using SPSS 16.0 software. Data were analyzed using one-way ANOVA followed by LSD test. $P$ values $<0.05$ were considered statistically significant.

\section{Results}

\section{Effect of RDN on cardiac hypertrophy and dysfunction}

At the end of the study period (10 weeks), 6, 6, 5 and 6 rats survived in Control, Sham, Carvedilol and RDN group, respectively. Cardiac structure and function was detected by echocardiography at week 5 and 10 . At 5 weeks post TAC, echocardiography revealed significantly increased diastole interventricular septal thickness (IVSd) and left ventricular posterior wall in diastole (LVPWd) in rats with TAC compared with Control, indicating that model had been sucessfully built. At 10 weeks post TAC, EF and FS were markedly increased in the RDN group rather than the Carvedilol group, compared with the Sham group. Also, IVSd and LVPWd were significantly increased in both RDN and Carvedilol group in comparison 
Table 1. Echocardiographic characteristics at Week 0,5 , and 10 . Num = number of rats; $\mathrm{EF}$ = ejection fraction; FS = fractional shortening; IVSd = diastole interventricular septal thickness; LVDd= left ventricular end diastolic dimension; LVPWd = left ventricular posterior wall in diastole. $* \mathrm{P}<0.05$ vs. Control group; \#P $<0.05$ vs. Sham group

\begin{tabular}{cccccccc}
\hline Group & Week & Num & EF $(\%)$ & FS(\%) & IVSd(mm) & LVDd $((\mathrm{mm})$ & LVPWd(mm) \\
\hline Control & 0 & 6 & $71.43 \pm 1.46$ & $41.64 \pm 1.25$ & $1.52 \pm 0.02$ & $6.33 \pm 0.11$ & $1.61 \pm 0.04$ \\
& 5 & 6 & $71.98 \pm 2.69$ & $42.82 \pm 2.24$ & $1.56 \pm 0.04$ & $7.60 \pm 0.03$ & $1.65 \pm 0.03$ \\
& 10 & 6 & $72.27 \pm 1.80$ & $43.16 \pm 1.49$ & $1.60 \pm 0.04$ & $8.26 \pm 0.24$ & $1.72 \pm 0.08$ \\
Sham & 0 & 12 & $71.13 \pm 0.59$ & $41.41 \pm 0.49$ & $1.52 \pm 0.02$ & $6.51 \pm 0.11$ & $1.57 \pm 0.03$ \\
& 5 & 10 & $69.40 \pm 1.94$ & $40.59 \pm 1.48$ & $1.94 \pm 0.03^{*}$ & $7.74 \pm 0.31$ & $2.01 \pm 0.03^{*}$ \\
& 10 & 6 & $58.57 \pm 4.22^{*}$ & $32.76 \pm 3.00^{*}$ & $1.66 \pm 0.08$ & $8.67 \pm 0.29$ & $1.85 \pm 0.10$ \\
Carvedilol & 0 & 12 & $71.45 \pm 1.35$ & $41.78 \pm 1.13$ & $1.52 \pm 0.03$ & $6.44 \pm 0.11$ & $1.62 \pm 0.03$ \\
& 5 & 9 & $67.24 \pm 2.62$ & $39.00 \pm 1.99$ & $1.95 \pm 0.03^{*}$ & $7.89 \pm 0.27$ & $2.06 \pm 0.04^{*}$ \\
& 10 & 5 & $65.80 \pm 2.75$ & $37.83 \pm 2.06$ & $1.86 \pm 0.05^{*}$ & $8.37 \pm 0.62$ & $2.02 \pm 0.09^{*}$ \\
RDN & 0 & 12 & $70.18 \pm 2.08$ & $40.97 \pm 1.77$ & $1.52 \pm 0.02$ & $6.54 \pm 0.08$ & $1.60 \pm 0.02$ \\
& 5 & 9 & $68.98 \pm 2.50$ & $40.37 \pm 1.96$ & $1.95 \pm 0.02^{*}$ & $7.71 \pm 0.18$ & $2.08 \pm 0.04^{*}$ \\
& 10 & 6 & $68.07 \pm 2.69 \#$ & $39.80 \pm 2.10^{*}$ & $1.96 \pm 0.10^{*} \#$ & $8.62 \pm 0.43$ & $2.06 \pm 0.12^{*}$ \\
\hline
\end{tabular}

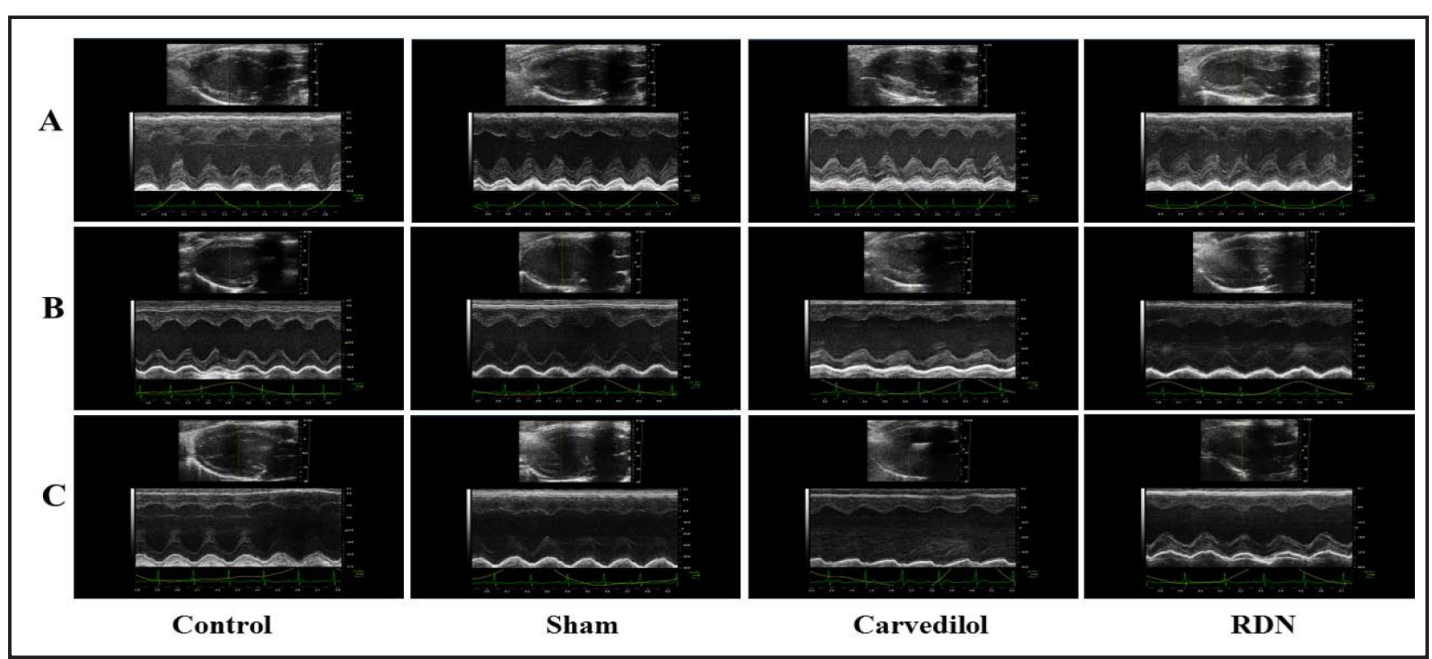

Fig. 1. RDN significantly improved cardiac hypertrophy and dysfunction. Representative tracings of echocardiography at week 0 (A), 5 (B), and 10 (C).

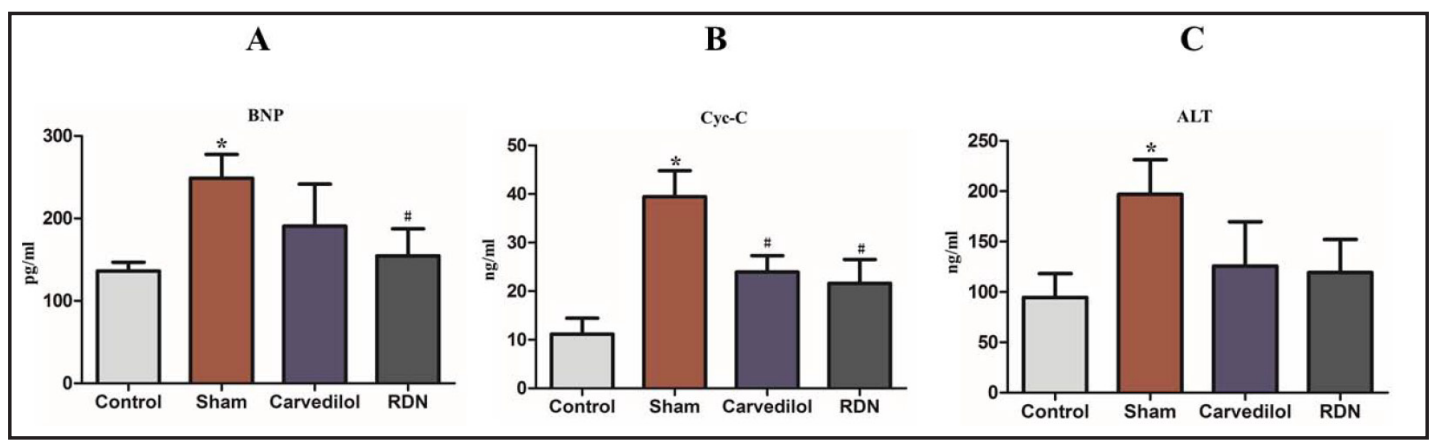

Fig. 2. RDN reduced the plasma levels of BNP and Cys-C. ELISA assay of (A) BNP, (B) Cys-C, (C) ALT. BNP: B-type natriuretic peptide; Cys-C: Cystatin C; ALT: Alanine Transaminase. (Group: Control n = 6; Sham n=6; Carvedilol $n=5$; RDN $n=6$ ). ${ }^{*} \mathrm{P}<0.05$ vs. Control group; $\# \mathrm{P}<0.05$ vs. Sham group.

with the Control group. However, there was no differences in LVDd among these four groups (Table 1 and Fig. 1).

\section{Effect of RDN on BNP, Cys-C and ALT}

Five weeks after RDN, the plasma levels of BNP, Cys-C and ALT were significantly increased in the Sham group but not in RDN or Carvedilol group, compared with the Control 


\section{Cellular Physiology Cell Physiol Biochem 2016;40:465-476 and Biochemistry Published online: November 25, $2016 \begin{aligned} & \text { DOI: 10.1159/000452561 } 2016 \text { The Author(s). Published by S. Karger AG, Base } \\ & \text { www.karger.com/cpb }\end{aligned}$

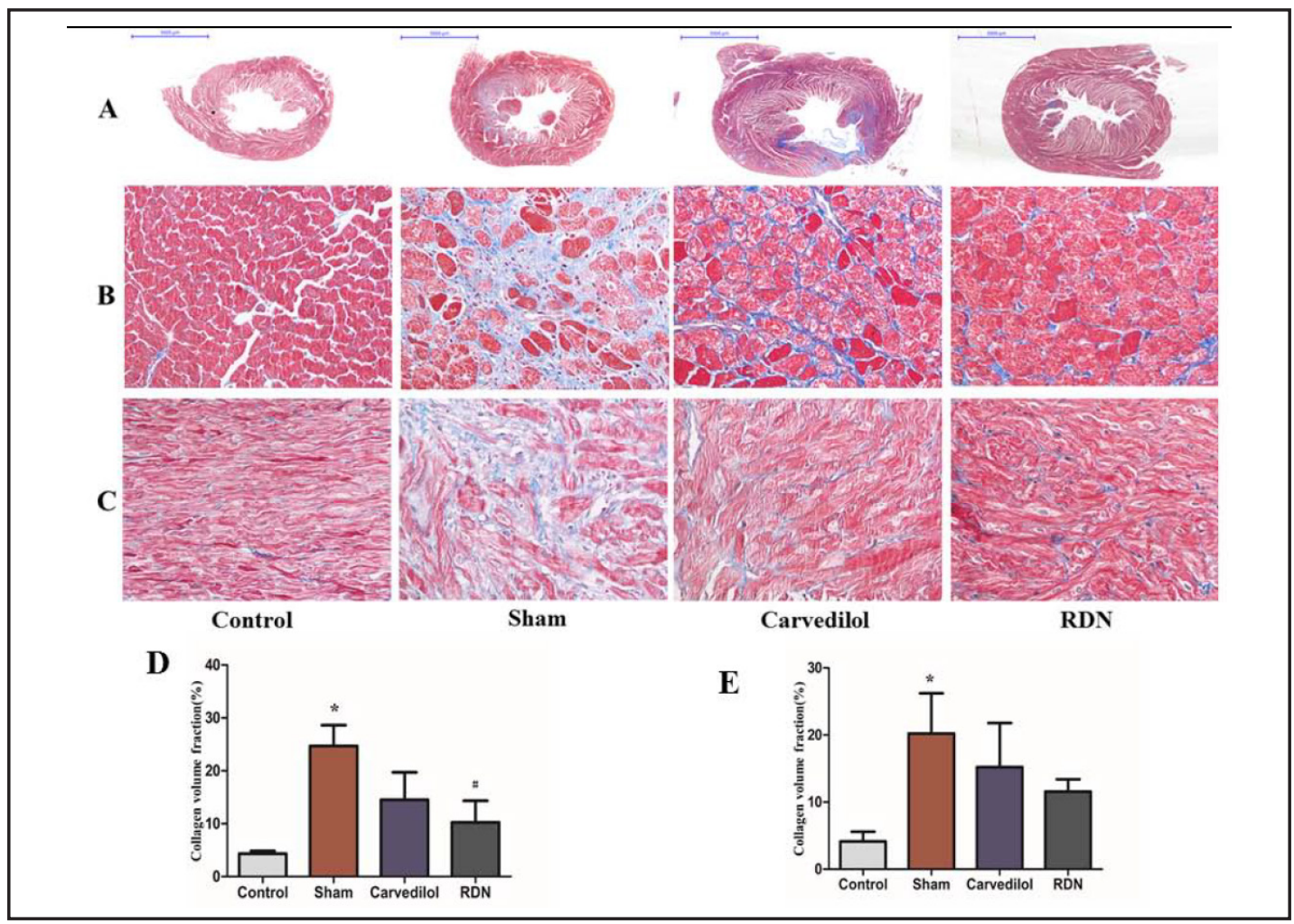

Fig. 3. Effect of RDN on fibrosis in LV and LA. Histological section of LV (A and B) and LA (C). Masson's Trichrome Staining (magnification $\times 5, \times 400$ and $\times 400$, respectively). The blue stands for fibrosis areas. CVF (LV fibrosis quantity) was significantly reduced in RDN group compared with Sham group (D). CVF (LA fibrosis quantity) was increased in Sham but not in RDN or Carvedilol group as compared with Control group (E). (Group: Control $n=6$; Sham $n=6$; Carvedilol $n=5$; RDN $n=6$ ). ${ }^{*} \mathrm{P}<0.05$ vs. Control group; $\# \mathrm{P}<0.05$ vs. Sham group.

group. Both RDN and Carvedilol reduced the Cys-C level while only RDN significantly reduced the BNP level in comparison with the Sham group (Fig. 2).

Effect of RDN on fibrosis in ventricle, atrium, kidneys and liver

Masson staining showed that $\mathrm{LV}$ and renal glomerular fibrosis were significantly reduced in the RDN group compared with the Sham group (Fig. 3 and 4). Both RDN and Carvedilol showed a decreasing trend of CVF in LA and renal tubulointerstitial compared with the Sham group (Fig. 3 and 4). In addition, both RDN and Carvedilol significantly reduced the extents of perivascular fibrosis of liver (Fig. 5).

\section{Effect of RDN on vascular remodeling}

The distal of the aortic constriction was examined. Masson staining showed that the media thickness of aorta was significantly reduced in both RDN and Carvedilol group compared with the Sham group. However, no significant differences were detected in the lumen diameter of aorta among all four groups. Also, both RDN and Carvedilol reduced the media/lumen, an index of vascular remodeling, in comparison with the Sham group (Fig. 6).

\section{Effect of RDN on blood pressure and heart rate}

Five weeks after RDN, the Sham group showed higher systolic BP than the Control group. Carvedilol but not RDN reduced the systolic BP compared with the Sham group (Fig. 7A). However, there was no significant difference in diastolic BP and HR among these four groups (Fig. 7B and 7C).

\section{KARGER}




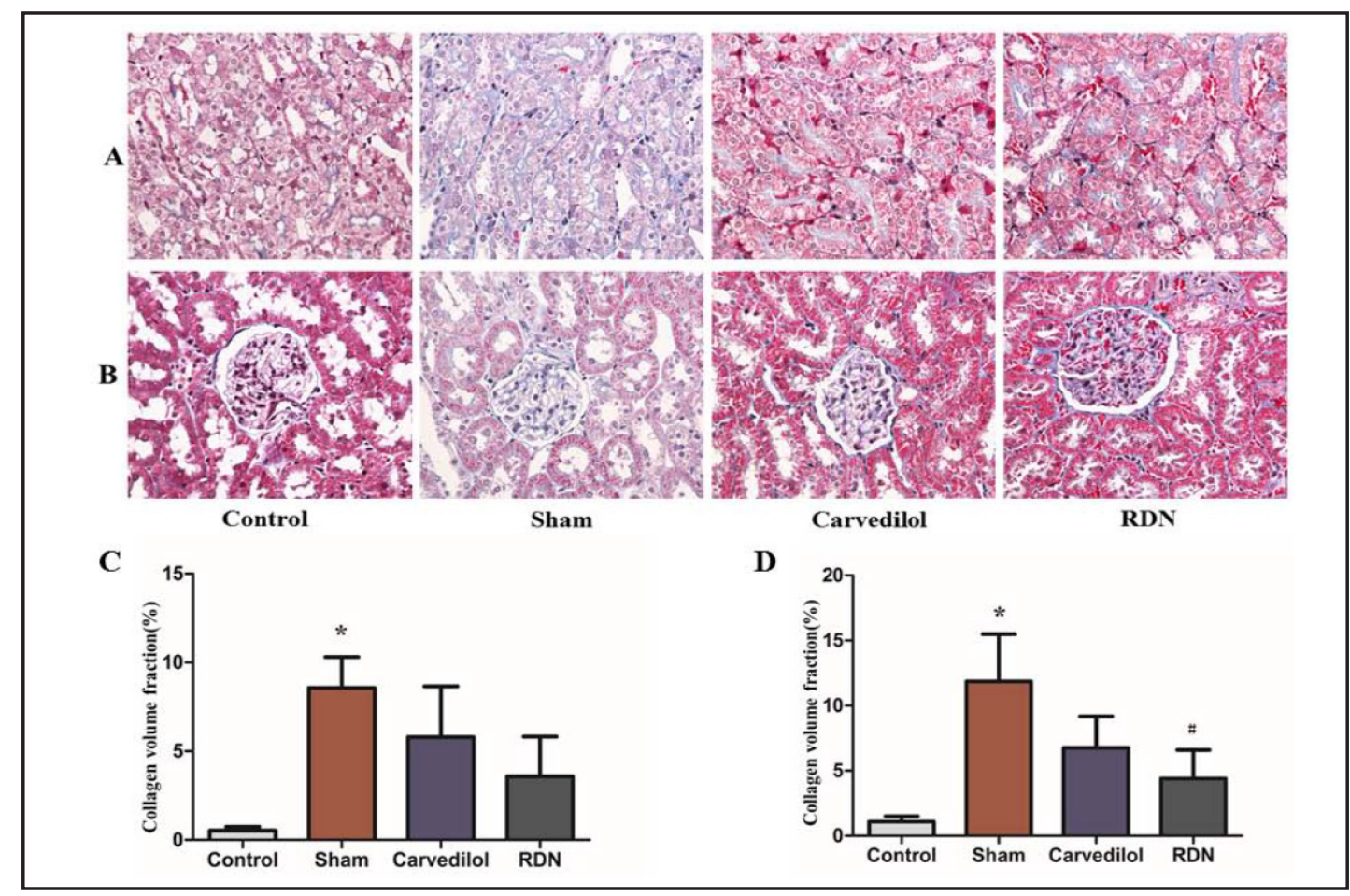

Fig. 4. Effect of RDN on renal fibrosis. Histological section of renal tubulointerstitial (A) and glomerular (B). Masson's Trichrome Staining (magnification $\times 400$ ). The blue stands for fibrosis areas. CVF (renal tubulointerstitial fibrosis quantity) was increased in Sham but not in RDN or Carvedilol group as compared with Control group (C). CVF (renal glomerular fibrosis quantity) was significantly reduced in RDN group compared with Sham group (D). (Group: Control $n=6$; Sham $n=6$; Carvedilol $n=5$; RDN $n=6$ ). *P $<0.05$ vs. Control group; \# P $<0.05$ vs. Sham group.

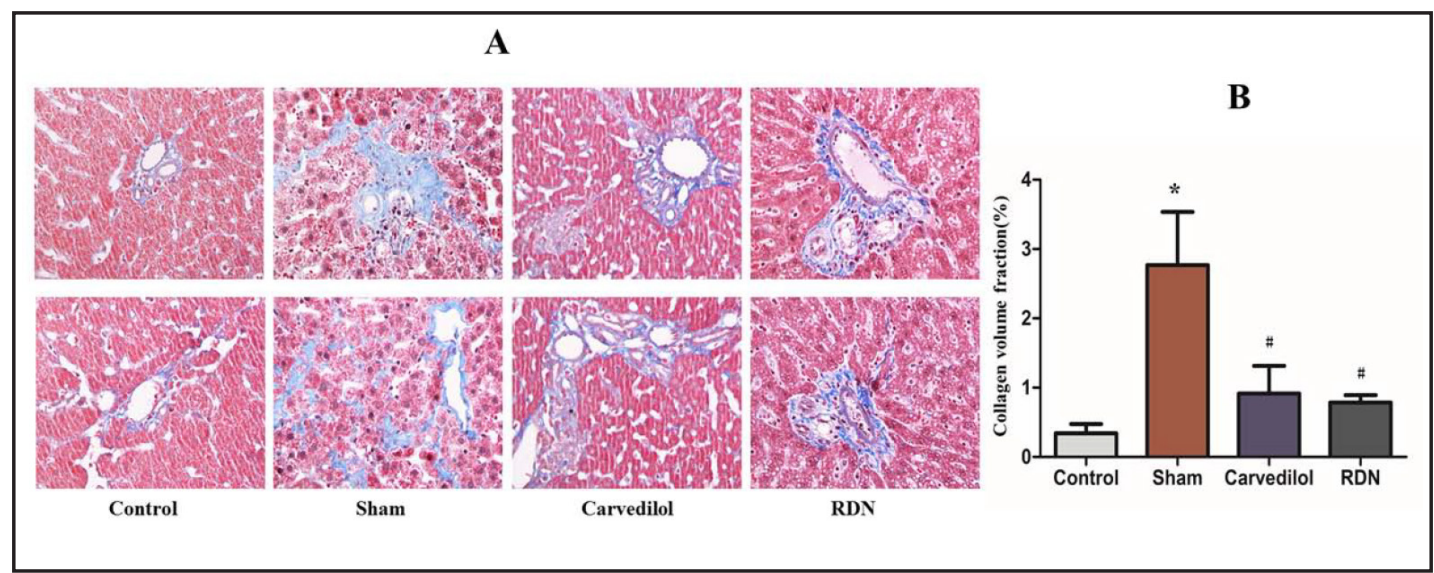

Fig. 5. RDN and Carvedilol significantly attenuated hepatic fibrosis. Histological section of liver (A). Masson's Trichrome Staining (magnification $\times 400$ ). The blue stands for fibrosis areas. CVF (hepatic perivascular fibrosis quantity) was significantly reduced in both RDN and Carvedilol group compared with Sham group (B). (Group: Control n=6; Sham n=6; Carvedilol n = 5; RDN n = 6). *P < 0.05 vs. Control group; \#P < 0.05 vs. Sham group.

\section{Efficacy of RDN Procedure}

Five weeks after RDN, tyrosine hydroxylase (TH) immunohistochemistry was analyzed for the activity of intrarenal sympathetic nerve fibers. The level of kidney TH expression

\section{KARGER}




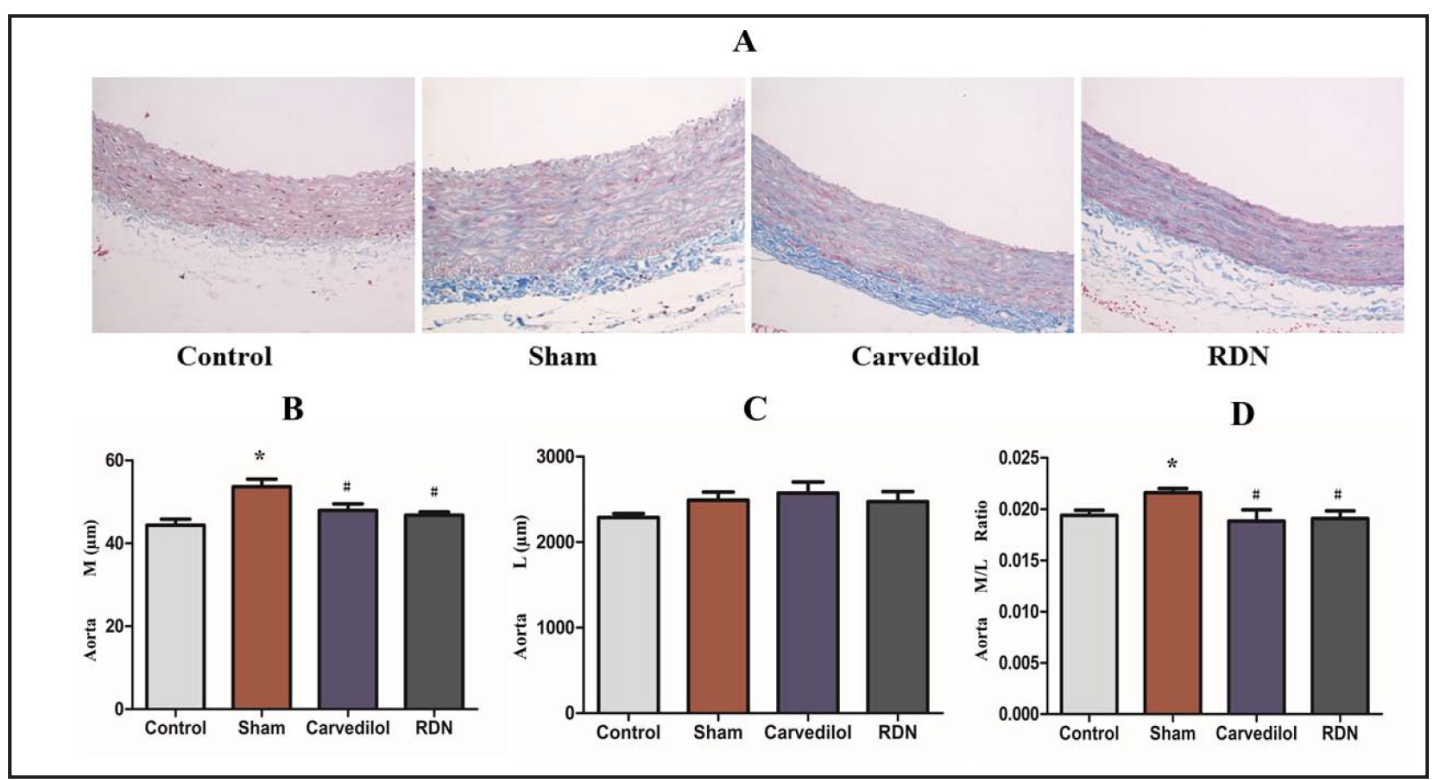

Fig. 6. RDN and Carvedilol significantly improved structural remodeling of the aorta. Histological section of aorta (A). Masson's Trichrome Staining (magnification $\times 200$ ). M (B) and M/L (D) were significantly reduced in both RDN and Carvedilol group compared with Sham group. Neither RDN nor Carvedilol significantly changed the L of aorta (C). M= media thickness; $L=$ lumen diameter. (Group: Control n = 6; Sham $n=6$; Carvedilol $n=5$; RDN $n=6$ ). ${ }^{*} \mathrm{P}<0.05$ vs. Control group; \#P $<0.05$ vs. Sham group.

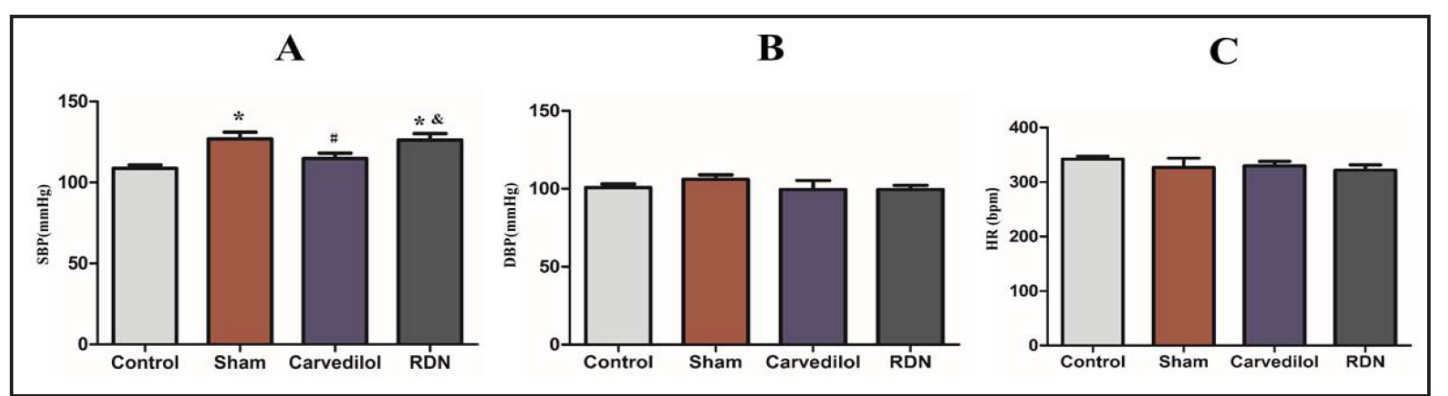

Fig. 7. RDN did not significantly reduced blood pressure and heart rate. SBP (A), DBP (B) and HR (C) at week 10. SBP: systolic blood pressure; DBP: diastolic pressure; HR: heart rate. (Group: Control n = 6; Sham $n=6$; Carvedilol $\mathrm{n}=5$; RDN $\mathrm{n}=6$ ). ${ }^{*} \mathrm{P}<0.05$ vs. Control group; $\# \mathrm{P}<0.05$ vs. Sham group; $\& \mathrm{P}<0.05$ vs. Carvedilol group.

was significantly reduced in the RDN group compared with the Sham group. Also, renal norepinephrine (NE) level was significantly decreased in both RDN and Carvedilol group in comparison with the Sham group. However, no significant differences were found in the plasma level of NE among these four groups (Fig. 8). These confirmed the completeness of RDN.

\section{Effect of RDN on TGF- $\beta 1$, PINP, PIIINP, Angiotensin II and Oxidative Stress}

Five weeks after RDN, the plasma levels of TGF- $\beta 1$, PINP, PIIINP, Ang II and MDA were significantly increased, T-SOD level was decreased in the Sham but not in RDN group, compared with the Control group. The level of PIIINP and Ang II decreased in RDN as compared with the Sham group. Both RDN and Carvedilol reduced TGF- $\beta 1$ concentration, and restored T-SOD level in comparison to the Sham group (Fig. 9).

\section{KARGER}




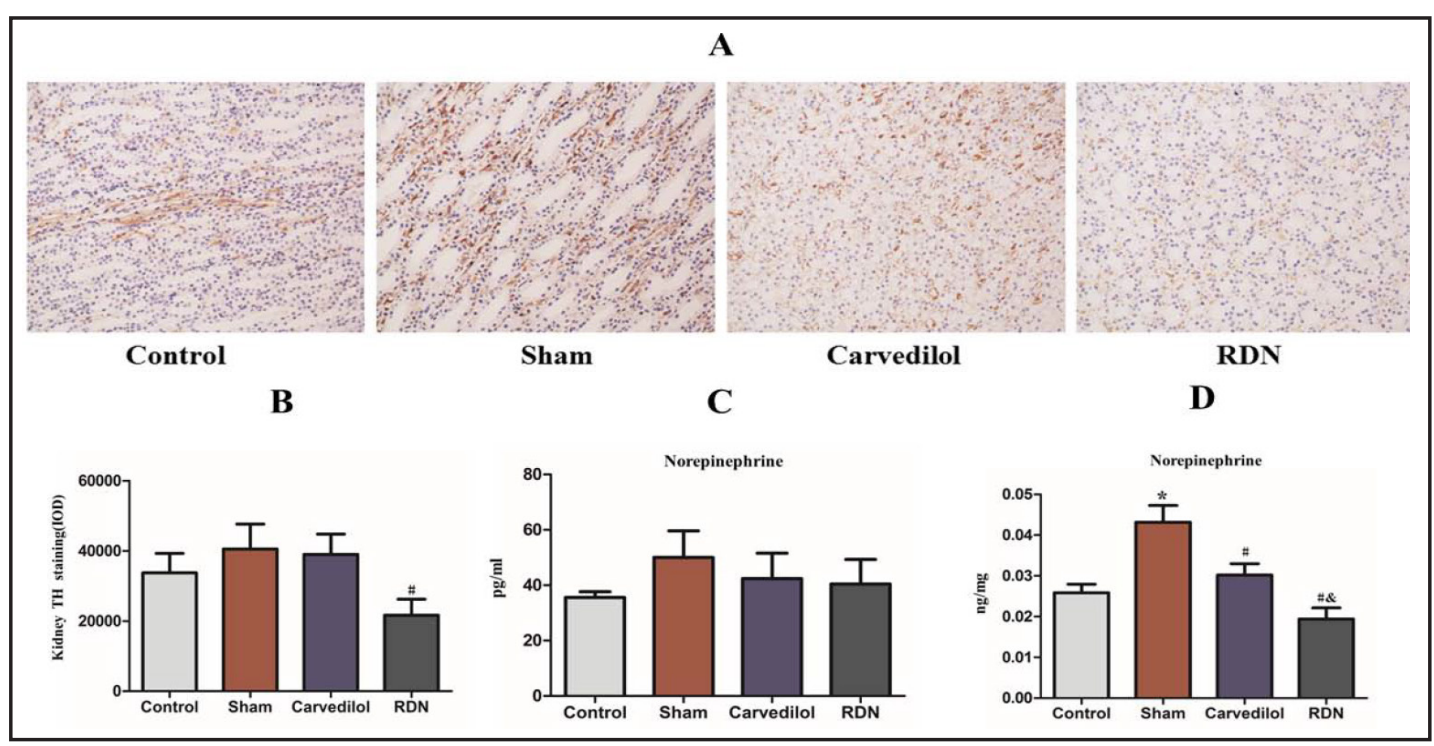

Fig. 8. RDN significantly reduced kidney expression of TH and the renal level of noradrenaline. (A) Representative images of immunohistochemical staining of renal TH protein expression (magnification $\times 400$ ). (B) Renal TH protein expression was significantly reduced in RDN group compared with Sham group (C) ELISA assay of plasma norepinephrine. Both RDN and Carvedilol had the tendency to reduce the plasma noradrenaline level as compared with Sham group. (D) ELISA assay of renal norepinephrine. Renal noradrenaline content was significantly reduced in RDN group in comparison with Sham group. TH: tyrosine hydroxylase; IOD: the integrated optical density per field. (Group: Control $n=6$; Sham $n=6$; Carvedilol $n=5$; RDN $n=6$ ). $* \mathrm{P}<0.05$ vs. Control group; \# $\mathrm{P}<0.05$ vs. Sham group; \& $\mathrm{P}<0.05$ vs. Carvedilol group.

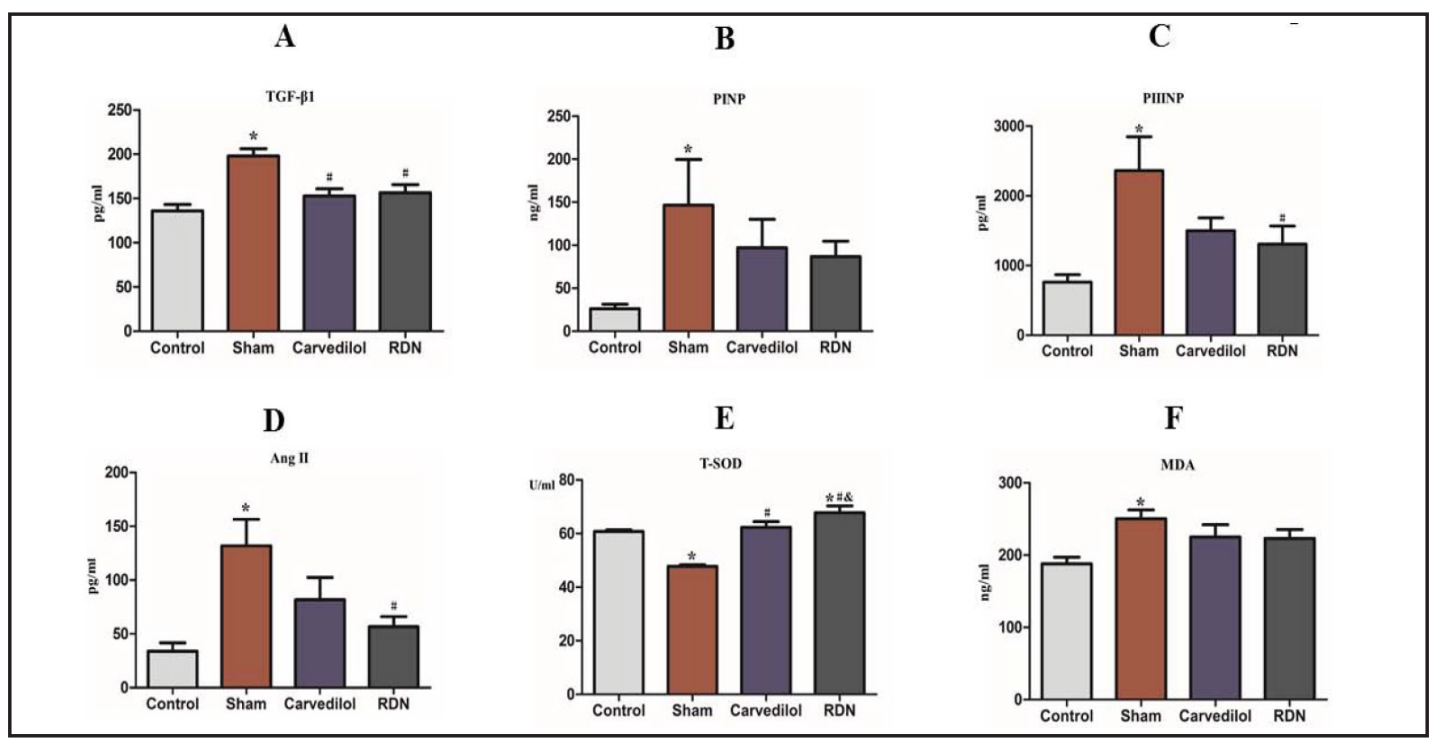

Fig. 9. Effects of RDN on TGF- $\beta 1$, PINP, PIIINP, Ang II and MDA as well as T-SOD levels. ELISA assay of (A) TGF- $\beta 1$, (B) PINP, (C) PIIINP, (D) Ang II, (E) T-SOD and (F) MDA. (Group: Control n=6; Sham n=6; Carvedilol $\mathrm{n}=5$; RDN $\mathrm{n}=6$ ). ${ }^{*} \mathrm{P}<0.05$ vs. Control group; $\# \mathrm{P}<0.05$ vs. Sham group; $\& \mathrm{P}<0.05$ vs. Carvedilol group.

\section{Discussion}

The major findings of this study are as follows: (1) RDN improves cardiac, renal and hepatic dysfunction in HF at the same time by inhibiting fibrosis; (2) RDN prevents cardiac, renal and hepatic fibrosis and improves vascular remodeling independent of BP and HR change; (3) RDN can directly inhibit RAAS system and oxidative stress. 
The levels of BNP, released predominantly from the ventricles, are related to degree of HF. Simultaneously, BNP level reveals a positive correlation with left ventricular end-diastolic pressure and with functional impairment as reflected by New York Heart Association (NYHA) class, meant that higher BNP level are associated with a higher mortality in HF [19]. Cystatin $\mathrm{C}$, reflecting glomerular filtration rate (GFR), is associated with an increase in all-cause mortality in HF [20, 21]. Abnormal liver function in HF is another predictor of increased mortality [22]. In our study, RDN could reduce BNP, Cystatin C and ALT levels, meant that RDN may reduce mortality in HF. What's more, these effects were comparable to carvedilol, a widely used drug in HF. The mechanism of RDN improving cardiac, renal and hepatic dysfunction as well as reducing mortality in HF may be associated with the inhibition of fibrosis.

Fibrosis is a common pathway to organ injury and failure [14]. Myocardial fibrosis causes cardiac systolic and diastolic dysfunction, cardiac arrhythmias and even sudden death. A study revealed that a $3 \%$ increase in extracellular volume fraction will increase $50 \%$ cardiovascular events [22]. Ventricular fibrosis can lead to LV dysfunction and ventricular arrhythmias [23] and atrial fibrosis plays an important role in occurrence and development of atrial fibrillation [24]. Atrial fibrosis has been an independent predictive factor of the cardiovascular events. Renal fibrosis, characterized by accumulation of extracellular matrix, is the common pathway for a variety of chronic renal disease that leads to end stage renal failure [25]. Hepatic fibrosis is the early reversible stage of cirrhosis and its most advanced stage is decompensated cirrhosis, along with various complications, arousing high morbidity and mortality [26]. Hypertension is a major risk factor for heart failure, cerebrovascular disease, myocardial infarction and peripheral artery disease. Simultaneously, hypertension is related to chronic vascular inflammation and remodeling, accelerating progressive vascular damage [27]. Moreover, vascular remodeling plays an important role in the pathogenesis and progression of atherosclerosis, hypertension and cardiovascular diseases [27]. PINP and PIIINP are regarded as the biomarkers of collagen types I and III synthesis as they are products during collagen production [2]. In our study, RDN attenuated LA, LV, tubulointerstitial, glomerular and hepatic fibrosis as well as improved vascular remodeling. All these were consistent with previous studies. In Dahl salt-sensitive hypertensive rats, RDN significantly reduced cardiac fibrosis[28]. Also, RDN markedly attenuated renal fibrosis in an ischemic renal injury model [29]. We recently assessed and compared impacts of RDN with losartan on cardiac and renal fibrosis in rats with pressure overload [30]. We found that RDN significantly prevented cardio-renal fibrosis through regulation of RAAS. The current study further demonstrated that RDN could attenuate cardiac, renal and hepatic fibrosis and improve vascular remodeling in HF at the same time. Differing from the previous study, we also compared RDN with carvedilol, a non-selective beta-blocker with alpha-blocker activity, which is often used to treat hypertension, heart failure and cirrhosis [31]. It can reduce myocardial fibrosis [32], renal fibrosis [33] and hepatic fibrosis [34]. In this study, we observed that RDN had non-inferior effect to carvedilol in protection from multi-organ fibrosis and vascular remodeling, involving prevention of oxidative stress in addition to RAAS.

The activation of the RAAS plays an important role in the development of organ fibrosis [3] and vascular remodeling[35]. Ang II, the main effector of RAAS, is regarded as a critical promoter of fibrogenesis. TGF- $\beta 1$, a kind of fibrogenic cytokines, is the key mediators of the pro-fibrotic effect of Ang II [36]. RDN significantly reduced the plasma levels of Ang II and TGF- $\beta 1$ in our study, which was consistent with previous results [17]. Carvedilol can also inhibit RAAS system and fibrogenic cytokines [33, 37]. RDN was non-inferior to carvedilol in inhibiting RAAS system in our study.

There is a crosstalk between RAAS and oxidative stress. Reactive oxygen species (ROS) are important mediators of the pro-fibrotic action of Ang II [38, 39]. Increased ROS production can lead to excess oxidative stress, which exhibits lipid peroxidation, protein oxidation, DNA damage and fibrosis $[39,40]$. Superoxide dismutase (SOD) plays a major role in antioxidant defense by catalyzing the dismutation process of superoxide anion into oxygen and hydrogen

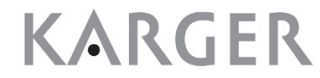


peroxide. Malonaldehyde (MDA), a product of lipid peroxidation, is regarded as an indicator of free radical activity and tissue damage [41]. In the failing heart, the expression of Ang II, TGF- $\beta 1$ and MDA increases, while the expression of SOD decreases [17, 42]. Elevated Ang II level can further increase the expression of TGF- $\beta 1$, which aggravates organ fibrosis and vascular remodeling. In our study, RDN lowered the plasma level of MDA and restored T-SOD level. Our findings are partly consistent with the study by Peleli et al. [43]. However, using a rat model of TAC induced HF, we compared RDN with carvedilol, a therapeutic drug for organ fibrosis and oxidative stress [34]. Simultaneously, improvements of multi-organ fibrosis and vascular remodeling were also observed in the present study.

There were several studies reporting of a subgroup of patients non-responding to RDN [44]. Similarly, no significant change in BP and HR following RDN was observed in our study. The small sample size may account for this. However, fibrosis and vascular remodeling were still improved. This is partly in line with previous clinical studies [44-46]. Therefore, it is plausible to find that RDN exerted its anti-fibrosis and anti-remodeling effects in a BP and HR independent manner in our study.

\section{Study limitation}

Some limitations of this study should be noted. First, TAC model can imitate the pressure overload induced by hypertension. Nonetheless, TAC model is not the same as essential hypertension. Second, we were unable to determine the optimal dose of carvedilol. Previous studies have shown that carvedilol has antioxidant effects in healthy subjects treated with small and moderate doses (6.25 - $25 \mathrm{mg} /$ day) [47]. We selected a common used dose in rats $(10 \mathrm{mg} / \mathrm{kg} /$ day) $[15,48]$. This dose of carvedilol therapy did not result in HR lowering in streptozotocin-induced diabetic rats [48]. Similarly, no HR-lowering effect of this dose of carvedilol was observed in this study. However, BP-lowering effect of carvedilol was detected, indicating the dose in our study had worked. Besides, we had no intention to promote the dose of carvedilol to clinical use. Last, further studies should investigate whether RDN can attenuate brain, lung and pancreas fibrosis.

\section{Conclusions}

RDN can attenuate multi-organ fibrosis and improve vascular remodeling independent of BP and HR change in TAC-induced cardiomyopathy. These effects of RDN may be associated with the direct inhibition of RAAS and oxidative stress.

\section{Acknowledgments}

This study was supported by the Natural Science Fund of Health Administration of Jiangsu Province (No. H201302).

We thank Dr. Lindsea C Booth, who is from the Florey Institute of Neuroscience and Mental Health, for her kind help in the language presentation.

\section{Disclosure Statement}

None.

\section{References}

1 Palano F, Paneni F, Sciarretta S, Tocci G, Volpe M: The progression from hypertension to congestive heart failure. Recenti Prog Med 2011;102:461-467.

2 Barison A, Grigoratos C, Todiere G, Aquaro GD: Myocardial interstitial remodelling in non-ischaemic dilated cardiomyopathy: insights from cardiovascular magnetic resonance. Heart Fail Rev 2015;20:731-749. 


\section{Cellular Physiology Cell Physiol Biochem 2016;40:465-476 and Biochemistry Published online: November 25, 2016 \begin{tabular}{l|l} 
DOI: 10.1159/000452561 & $\begin{array}{l}\text { C } 2016 \text { The Author(s). Published by S. Karger AG, Basel } \\
\text { www.karger.com/cpb }\end{array}$
\end{tabular} \\ Wang et al.: Renal Denervation and Multi-Organ Fibrosis}

3 Husain-Syed F, McCullough PA, Birk HW, Renker M, Brocca A, Seeger W, Ronco C: Cardio-Pulmonary-Renal Interactions: A Multidisciplinary Approach. J Am Coll Cardiol 2015;65:2433-2448.

4 Herzer K, Kneiseler G, Bechmann LP, Post F, Schlattjan M, Sowa JP, Neumann T, Marggraf G, Erbel R, Gerken G, Canbay A, Jochum C: Onset of heart failure determines the hepatic cell death pattern. Ann Hepatol 2011;10:174-179.

5 Li X, Hassoun HT, Santora R, Rabb H: Organ crosstalk: the role of the kidney. Curr Opin Crit Care 2009;15:481-487.

6 Zhang F, Dang Y, Li Y, Hao Q, Li R, Qi X: Cardiac Contractility Modulation Attenuate Myocardial Fibrosis by Inhibiting TGF-beta1/Smad3 Signaling Pathway in a Rabbit Model of Chronic Heart Failure. Cell Physiol Biochem 2016;39:294-302.

7 Schuetze KB, McKinsey TA, Long CS: Targeting cardiac fibroblasts to treat fibrosis of the heart: focus on HDACs. J Mol Cell Cardiol 2014;70:100-107.

8 Munoz-Felix JM, Gonzalez-Nunez M, Martinez-Salgado C, Lopez-Novoa JM: TGF-beta/BMP proteins as therapeutic targets in renal fibrosis. Where have we arrived after 25 years of trials and tribulations? Pharmacol Ther 2015;156:44-58.

9 Arriazu E, Ge X, Leung TM, Magdaleno F, Lopategi A, Lu Y, Kitamura N, Urtasun R, Theise N, Antoine DJ, Nieto N: Signalling via the osteopontin and high mobility group box-1 axis drives the fibrogenic response to liver injury. Gut DOI:10.1136/gutjnl-2015-310752.

10 Yang J, Chen YN, Xu ZX, Mou Y, Zheng LR: Alteration of RhoA Prenylation Ameliorates Cardiac and Vascular Remodeling in Spontaneously Hypertensive Rats. Cell Physiol Biochem 2016;39:229-241.

11 Xiao L, Kirabo A, Wu J, Saleh MA, Zhu L, Wang F, Takahashi T, Loperena R, Foss JD, Mernaugh RL, Chen W, Roberts J, 2nd, Osborn JW, Itani HA, Harrison DG: Renal Denervation Prevents Immune Cell Activation and Renal Inflammation in Angiotensin II-Induced Hypertension. Circ Res 2015;117:547-557.

12 Ukena C, Mahfoud F, Spies A, Kindermann I, Linz D, Cremers B, Laufs U, Neuberger HR, Bohm M: Effects of renal sympathetic denervation on heart rate and atrioventricular conduction in patients with resistant hypertension. Int J Cardiol 2013;167:2846-2851.

13 Schirmer SH, Sayed MM, Reil JC, Ukena C, Linz D, Kindermann M, Laufs U, Mahfoud F, Bohm M: Improvements in left ventricular hypertrophy and diastolic function following renal denervation: effects beyond blood pressure and heart rate reduction. J Am Coll Cardiol 2014;63:1916-1923.

14 Rockey DC, Bell PD, Hill JA: Fibrosis--a common pathway to organ injury and failure. N Engl J Med 2015;372:1138-1149.

15 Hamdy N, El-Demerdash E: New therapeutic aspect for carvedilol: antifibrotic effects of carvedilol in chronic carbon tetrachloride-induced liver damage. Toxicol Appl Pharmacol 2012;261:292-299.

16 Ji XB, Li XR, Hao D, Sun Q Zhou Y, Wen P, Dai CS, Yang JW: Inhibition of Uncoupling Protein 2 Attenuates Cardiac Hypertrophy Induced by Transverse Aortic Constriction in Mice. Cell Physiol Biochem 2015;36:1688-1698.

17 Liu Q, Zhang Q, Wang K, Wang S, Lu D, Li Z, Geng J, Fang P, Wang Y, Shan Q: Renal Denervation Findings on Cardiac and Renal Fibrosis in Rats with Isoproterenol Induced Cardiomyopathy. Sci Rep 2015;5:18582.

18 Bagnost T, Ma L, da Silva RF, Rezakhaniha R, Houdayer C, Stergiopulos N, Andre C, Guillaume Y, Berthelot A, Demougeot C: Cardiovascular effects of arginase inhibition in spontaneously hypertensive rats with fully developed hypertension. Cardiovasc Res 2010;87:569-577.

19 Suzuki T, Lyon A, Saggar R, Heaney LM, Aizawa K, Cittadini A, Mauro C, Citro R, Limongelli G, Ferrara F, Vriz O, Morley-Smith A, Calabro P, Bossone E: Biomarkers of acute cardiovascular and pulmonary diseases. Eur Heart J Acute Cardiovasc Care DOI:10.1177/2048872616652309.

20 Carrasco-Sanchez FJ, Perez-Calvo JI, Morales-Rull JL, Galisteo-Almeda L, Paez-Rubio I, Baron-Franco B, Aguayo-Canela M, Pujol-De la llave E: Heart failure mortality according to acute variations in N-terminal pro B-type natriuretic peptide and cystatin C levels. J Cardiovasc Med (Hagerstown) 2014;15:115-121.

21 Herget-Rosenthal S, Trabold S, Pietruck F, Holtmann M, Philipp T, Kribben A: Cystatin C: efficacy as screening test for reduced glomerular filtration rate. Am J Nephrol 2000;20:97-102.

22 Wong TC, Piehler K, Meier CG, Testa SM, Klock AM, Aneizi AA, Shakesprere J, Kellman P, Shroff SG, Schwartzman DS, Mulukutla SR, Simon MA, Schelbert EB: Association between extracellular matrix expansion quantified by cardiovascular magnetic resonance and short-term mortality. Circulation 2012;126:1206-1216.

23 Iles L, Pfluger H, Lefkovits L, Butler MJ, Kistler PM, Kaye DM, Taylor AJ: Myocardial fibrosis predicts appropriate device therapy in patients with implantable cardioverter-defibrillators for primary prevention of sudden cardiac death. J Am Coll Cardiol 2011;57:821-828.

24 Zahid S, Cochet H, Boyle PM, Schwarz EL, Whyte KN, Vigmond EJ, Dubois R, Hocini M, Haissaguerre M, Jais P, Trayanova NA: Patient-derived models link reentrant driver localization in atrial fibrillation to fibrosis spatial pattern. Cardiovasc Res 2016;110:443-454. 


\section{Cellular Physiology Cell Physiol Biochem 2016;40:465-476 and Biochemistry Published \begin{tabular}{l|l} 
DOI: 10.1159/000452561 & $\begin{array}{l}\text { C } 2016 \text { The Author(s). Published by S. Karger AG, Basel } \\
\text { www.karger.com/cpb }\end{array}$
\end{tabular} \\ Wang et al.: Renal Denervation and Multi-Organ Fibrosis}

25 Strutz F, Zeisberg M: Renal fibroblasts and myofibroblasts in chronic kidney disease. J Am Soc Nephrol 2006;17:2992-2998.

26 Ismail MH, Pinzani M: Reversal of liver fibrosis. Saudi J Gastroenterol 2009;15:72.

27 Dorr O, Liebetrau C, Mollmann H, Mahfoud F, Ewen S, Gaede L, Troidl C, Hoffmann J, Busch N, Laux G, Wiebe J, Bauer T, Hamm C, Nef H: Beneficial effects of renal sympathetic denervation on cardiovascular inflammation and remodeling in essential hypertension. Clin Res Cardiol 2015;104:175-184.

28 Watanabe H, Iwanaga Y, Miyaji Y, Yamamoto H, Miyazaki S: Renal denervation mitigates cardiac remodeling and renal damage in Dahl rats: a comparison with $\beta$-receptor blockade. Hypertens Res 2016;39:217-226.

29 Kim J, Padanilam BJ: Renal denervation prevents long-term sequelae of ischemic renal injury. Kidney Int 2015;87:350-358.

30 Liu Q, Lu D, Wang S, Wang K, Zhang Q, Wang Y, Fang P, Li Z, Geng J, Shan Q: Renal denervation significantly attenuates cardiorenal fibrosis in rats with sustained pressure overload. J Am Soc Hypertens 2016;10:587596.e584.

31 Reiberger T, Ulbrich G, Ferlitsch A, Payer BA, Schwabl P, Pinter M, Heinisch BB, Trauner M, Kramer L, Peck-Radosavljevic M: Carvedilol for primary prophylaxis of variceal bleeding in cirrhotic patients with haemodynamic non-response to propranolol. Gut 2013;62:1634-1641.

32 Chen J, Huang C, Zhang B, Huang Q Chen J, Xu L: The effects of carvedilol on cardiac structural remodeling: the role of endogenous nitric oxide in the activity of carvedilol. Mol Med Rep 2013;7:1155-1158.

33 Wong VY, Laping NJ, Nelson AH, Contino LC, Olson BA, Gygielko E, Campbell WG, Jr., Barone F, Brooks DP: Renoprotective effects of carvedilol in hypertensive-stroke prone rats may involve inhibition of TGF beta expression. Br J Pharmacol 2001;134:977-984.

34 Araujo Junior RF, Garcia VB, Leitao RF, Brito GA, Miguel Ede C, Guedes PM, de Araujo AA: Carvedilol Improves Inflammatory Response, Oxidative Stress and Fibrosis in the Alcohol-Induced Liver Injury in Rats by Regulating Kuppfer Cells and Hepatic Stellate Cells. PLoS One 2016;11:e0148868.

35 Pacurari M, Kafoury R, Tchounwou PB, Ndebele K: The Renin-Angiotensin-aldosterone system in vascular inflammation and remodeling. Int J Inflam 2014;2014:689360.

36 Ruiz-Ortega M, Rodriguez-Vita J, Sanchez-Lopez E, Carvajal G, Egido J: TGF-beta signaling in vascular fibrosis. Cardiovasc Res 2007;74:196-206.

37 Abdulla MH, Sattar MA, Abdullah NA, Johns EJ: The effect of losartan and carvedilol on renal haemodynamics and altered metabolism in fructose-fed Sprague-Dawley rats. J Physiol Biochem 2012;68:353-363.

38 Macconi D, Remuzzi G, Benigni A: Key fibrogenic mediators: old players. Renin-angiotensin system. Kidney Int Suppl (2011) 2014;4:58-64.

39 Munzel T, Gori T, Keaney JF, Jr., Maack C, Daiber A: Pathophysiological role of oxidative stress in systolic and diastolic heart failure and its therapeutic implications. Eur Heart J 2015;36:2555-2564.

40 Gutterman DD, Chabowski DS, Kadlec AO, Durand MJ, Freed JK, Ait-Aissa K, Beyer AM: The Human Microcirculation: Regulation of Flow and Beyond. Circ Res 2016;118:157-172.

41 Liu FJ, Li SX, Huang BQ, Zheng FY, Huang XG: Effect of excessive CO2 on physiological functions in coastal diatom. Sci Rep 2016;6:21694.

42 Mondal NK, Sorensen EN, Pham SM, Koenig SC, Griffith BP, Slaughter MS, Wu ZJ: Systemic Inflammatory Response Syndrome in End-Stage Heart Failure Patients Following Continuous-Flow Left Ventricular Assist Device Implantation: Differences in Plasma Redox Status and Leukocyte Activation. Artif Organs 2016;40:434-443.

43 Peleli M, Al-Mashhadi A, Yang T, Larsson E, Wahlin N, Jensen BL, AE GP, Carlstrom M: Renal denervation attenuates NADPH oxidase-mediated oxidative stress and hypertension in rats with hydronephrosis. Am J Physiol Renal Physiol 2016;310:F43-56.

44 Brandt MC, Mahfoud F, Reda S, Schirmer SH, Erdmann E, Bohm M, Hoppe UC: Renal sympathetic denervation reduces left ventricular hypertrophy and improves cardiac function in patients with resistant hypertension. J Am Coll Cardiol 2012;59:901-909.

45 Schirmer SH, Sayed MM, Reil JC, Lavall D, Ukena C, Linz D, Mahfoud F, Bohm M: Atrial Remodeling Following Catheter-Based Renal Denervation Occurs in a Blood Pressure- and Heart Rate-Independent Manner. JACC Cardiovasc Interv 2015;8:972-980.

46 Lu D, Wang K, Liu Q, Wang S, Zhang Q, Shan Q: Reductions of left ventricular mass and atrial size following renal denervation: a meta-analysis. Clin Res Cardiol 2016;105:648-656.

47 Dandona P, Karne R, Ghanim H, Hamouda W, Aljada A, Magsino CH, Jr.: Carvedilol inhibits reactive oxygen species generation by leukocytes and oxidative damage to amino acids. Circulation 2000;101:122-124.

48 Fu GS, Huang H, Chen F, Wang HP, Qian LB, Ke XY, Xia Q: Carvedilol ameliorates endothelial dysfunction in streptozotocin-induced diabetic rats. Eur J Pharmacol 2007;567:223-230. 